\title{
The Ion Produced Transverse Instabilities in SRRC Storage Ring
}

\author{
J. C. Lee and M. H. Wang \\ SRRC, Hsinchu Science-Based Industrial Park, Hsinchu, Taiwan, ROC
}

\begin{abstract}
The ions captured by the electron beam potential can introduce transverse instabilities and deteriorate the beam quality. For SRRC storage ring, the transverse instabilities due to trapped ions are found since commissioning and affect the beam performance. In this paper, we summarize the measurements of transverse instabilities which related to the ions. The efforts used to reduced the instabilities are also presented in the paper.
\end{abstract}

\section{INTRODUCTION}

The SRRC storage ring is a dedicated synchrotron light source in the VUV and soft X-ray. It was design and operated at $1.3 \mathrm{GeV}$. While it was upgraded to $1.5 \mathrm{GeV}$ in 1996 to increase the emitted photon energy and the beam performance. Since commissioning of the ring, the transverse instabilities were found occasionally. The trapped ions effect was one of the suspicious. Since then measurements were performed to study these effects. They includes the general investigations from the conventional ion points of view, the tried cures and the venting $\mathrm{H}_{2}$ gas studies. For effects of fast beam ion instability, the measurements are very few due to instrument issues. Since the dust trapping has relations to the ions, this phenomenon is also discussed in the paper.

From the commissioning phase till now, many efforts were down to improve the ring performance. Among them, the energy upgrade and the improvement in vacuum pressure reduce the instabilities by reducing the driven force from ions. In contrast to the reduction of ion driven force, the systems such as damper, beam shaking and clean electrode etc, eliminate the instability by interfering the beam. There are the third kind methods such as empty gap in the bunch train and strong chromaticities. Most of the curing methods would be discussed in the paper.

\section{DUST TRAPPING}

During the commissioning of the ring, the vacuum pressure was gradually pumped down as the increasing of beam dosage. While at that time the beam current and lifetime had a sudden jump occasionally. In the latter operation the sudden beam lost became not obvious while the lifetime still had a dip. This effect was suspicious from the dust trapping phenomenon. To identify this assumption the bremsstrahlung emitted from the beam was measured by Cherenkov counter. As the beam encountered the massive dust, electron beam will interact with the protons in the dust such that the beam would be bent and accelerated to emit bremsstrahlung. Figure 1 shows the consistence from the dip in beam lifetime and the sudden increasing in counter readings. The dust trapping phenomenon was verified at commissioning phase. While this phenomenon disappeared as the vacuum conditions getting better.

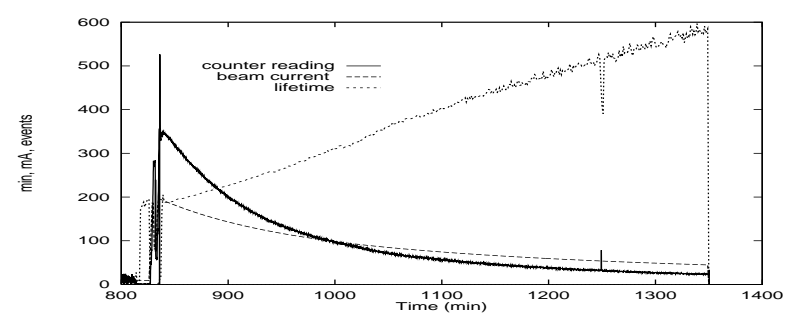

Figure 1: Indication of dust trapping from the dip of beam lifetime and the corresponding reading increasing in Cherenkov counter.

\section{OBSERVATIONS IN CONVENTIONAL ION EFFECTS}

In the commissioning phase the transverse instabilities were found from the pickup signal of stripline electrode, as shown in figure 2 for a all of buckets uniformly filled pattern. The spontaneous betatron sidebands in horizontal and vertical indicate the instabilities. The longitudinal sidebands is accompanied with the peaks of revolution and betatron sidebands. The emitted photon beam was observed as the instrument was ready. It was found the beam size pulsated as there were the spontaneous betatron sidebands. Many efforts were tried to understand these instabilities. The ion trapping effect was one of the suspicious. It was found a very big empty gap in the filling pattern can reduce and dismiss the oscillation peaks. A big enough empty gap in the filled bunch train is the typical curing method for the ion trapping phenomenon. Since then the ion trapping phenomenon was studied and reported[1].

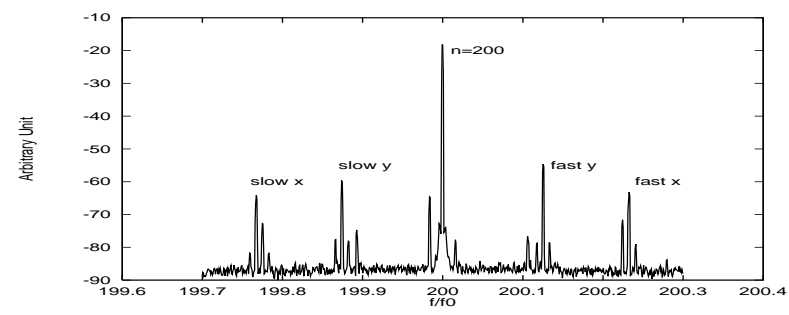

Figure 2: Spontaneous oscillation peaks at the revolution of $n=200$ for a all of buckets filled pattern.

From the residual gas analyzer data, $\mathrm{H}_{2}$ and $\mathrm{CO}$ are the 
than $90 \%$ population and CO only takes about $4 \%$. While since the ionization rate for $\mathrm{CO}$ is larger than that for $\mathrm{H}_{2}$, these two species were suspicious for the ion instabilities at the beginning. From the experimental results of how big of the empty gap to avoid the spontaneous transverse peaks and from theoretic prediction, the $\mathrm{H}_{2}$ ion is the major contributor for the ion instabilities. This is also verified by the venting $\mathrm{H}_{2}$ gas experiments recently ${ }^{[2]}$.

The trapped ions will introduce an additional quadrupole force on beam, which implies the lattice will be perturbed a little bit such that the betatron tune will be shifted by the ion force. This was basically confirmed by the measurements of tune versus different empty gap.

For more understandings, a pattern was filled to have the possibility to trap ions and chromaticities were applied to damp the instabilities at the normal operation pressure. The ion driven force was then increased by the trapped ion density, which is increased from the increasing of vacuum pressure by turning off some of the pumps. It was found that the vertical betatron sideband showed up first at around 12 ntorr and then the horizontal sideband at higher vacuum pressure. It was also found the transverse betatron peaks have threshold current limits. The current limit for the vertical is smaller than that for horizontal.

\section{THE TRIED REMEDY METHODS}

\subsection{Empty gap and chromaticity}

For the possible cures the empty gap in the filling pattern is an easy method to be applied. While the gap needed to avoid trapping was large, around 120 or bigger from the $200 \mathrm{RF}$ buckets at $250 \mathrm{~mA}$. The beam lifetime became small since beam population per bunch getting large in big empty gap operation. The compromise was the small empty gap, say 20 to 40 buckets, with the damping mechanism from chromaticities. The chromaticities were found very effective to damp the transverse peaks in both planes. For big gap operation only small chromaticities were needed to damp transverse peaks. If the gap was big enough, there were no transverse peaks even at zero chromaticities. While the chromaticities should be increased up to +6.5 for all of the buckets filled one at $1.3 \mathrm{GeV}$. The bad effect of appling strong chromaticities is that it increases the sextupole strength such that the beam lifetime and the dynamic aperture reduced.

\subsection{Clean electrode}

The clean electrodes were also tried to remove ions. One button type electron beam position monitor and one $15 \mathrm{~cm}$ long stripline electrode were modified for this studies. The remedy is clearly shown form the reduction of tune and tune spread as the clean voltage applied. While the clean ability is not enough for only two electrodes .
The beam shaken at the betatron sidebands was effective to remove the instabilities from ions ${ }^{[3]}$. In this studies one $50 \mathrm{~cm}$ long stripline electrode was modified to input the power to shake the beam periodically. As the beam was proper shaken the transverse peaks were dismissed. The beam pulsation disappeared and the beam size reduced. While the lifetime increased for around 2 hours at $1.3 \mathrm{GeV}$. The studies also show the shaken frequency is not only effective at coherent betatron sidebands but also at the betatron sidebands of which no coherent oscillation peaks showed up. The shaking frequency was applied by sweeping a range from 0 to $34 \mathrm{MHz}$. It was found the frequency of betatron sidebands within this range are almost effective to damp the instabilities. But it is more effective for the sidebands of coherent oscillation. It was also found that slow betatron sidebands were more effective than fast sidebands.

Though the goal of longitudinal beam modulation by the second synchrotron sideband is try to cure the longitudinal instabilities $^{[4]}$, the transverse oscillation peaks are also reduced or damped, dependent on the operation conditions. The pulsation and beam size are reduced in the modulation process. These phenomena are similar to that of the transverse shaking at the betatron sidebands.

\subsection{Other improvements}

These improvements include the beam energy upgrade from $1.3 \mathrm{GeV}$ to $1.5 \mathrm{GeV}^{[5]}$ and the improvement in vacuum $^{[6]}$. Though the authors were not engaged in these two improvements, while the impacts were considerable. Increased beam energy will benefit beam stabilities from reducing the ion driven force as well as the other driven sources such as the force of wake field etc. The improvement on vacuum pressure can also reduce the ion driven force by reducing the trapped ion density. Another method of reducing ion driven force is to increase the beam size. In the past year a high emittance lattice was operated for the users with the diluted beam size to reduce the instabilities. This can be taken as the example for increasing beam size. While the emitted photon intensity is reduced in this diluted beam size operation.

\section{OBSERVATION IN FAST BEAM ION EFFECTS}

The fast beam ion effect was proposed ${ }^{[7]}$ and studied ${ }^{[8,9]}$ in other laboratories. Due to the instrument issue, we can't observe the bunch by bunch motion directly. For the undirect measurement, the scraper was used to scrape the beam with a $75 / 200$ filled pattern at $40 \mathrm{~mA}$ to avoid conventional ion trapping. Before scraping there were some of missing bunches within the bunch train and the population in head bunches is smaller than that of the tail bunches. While the tail bunches are roughly uniformly filled. This experiment was down as the vacuum chamber were replaced in January 1998. The local pressure of the replacing section was 
ter scraping is shown in figure 3 for the less population in the tail of the bunch train, which indicates the increasing of instability along the train. This result is similar to that in $\mathrm{ALS}^{[6]}$.

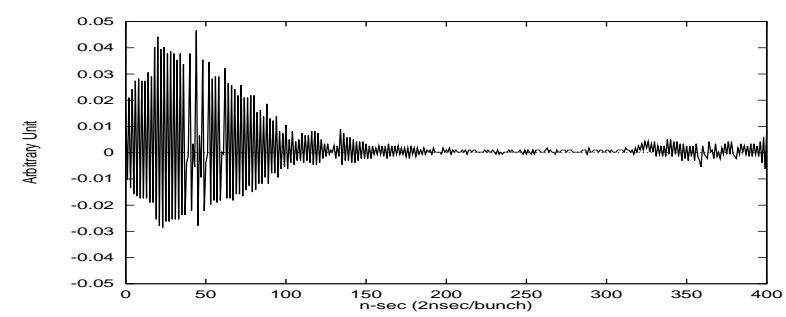

Figure 3: Beam population along the bunch train after scraping the beam.

\section{VENTING $\mathrm{H}_{2}$ GAS EXPERIMENT}

Since the $\mathrm{H}_{2}$ ion is the dominate species in the ring and would cause instability, the venting $\mathrm{H}_{2}$ gas experiments were performed at $1.5 \mathrm{GeV}$ recently to increase the $\mathrm{H}_{2}$ density in different cases ${ }^{[3]}$. From the studied results the $\mathrm{H}_{2}$ ion is confirmed to be the dominate source again for the ion instability. The coherent peaks from the button electrode is identified to be the $\mathrm{H}_{2}$ ion oscillation frequency as the gap is not big enough or as the local pressure of $\mathrm{H}_{2}$ increased up to around 60 ntorr. The coherent peaks seem have periodic structure with the period of revolution. As the vacuum pressure as high up to 500 ntorr, additional peaks show up besides the coherent frequency peak of $\mathrm{H}_{2}$ ion even at a 75/200 buckets filled pattern of which no ions could be trapped from the conventional theory prediction and no coherent oscillation before and after venting $\mathrm{H}_{2}$ gas. Similar spectrum, as shown in figure 5, was found for a all of buckets filled pattern at $1.3 \mathrm{GeV}$ in the normal operation vacuum pressure. Compared above two results, the coherent peaks have a little different due to different operation conditions. While ion driven force is increased for these two cases, which implies that the ion driven force could be the key point for the instability. Increasing the vacuum pressure, reducing the beam energy and operated with the pattern of all of buckets filled arrive the same destinate. That is increasing the driven force from ions. The known theories can't fully explain these additional peaks. Therefore the 'impedance like' idea for the ion force is introduced to explain these phenomena. While more works will be done in the future, including the theory and experiments.

\section{DISCUSSION}

The ion produced instabilities were investigated in the SRRC storage ring. From the studied results the driven force from ions is the key for the instabilities. As the empty gap is not big enough in the bunch train the trapped ions would cause instability. While if the empty gap is big enough to reduce the trapping and the driven force was de-

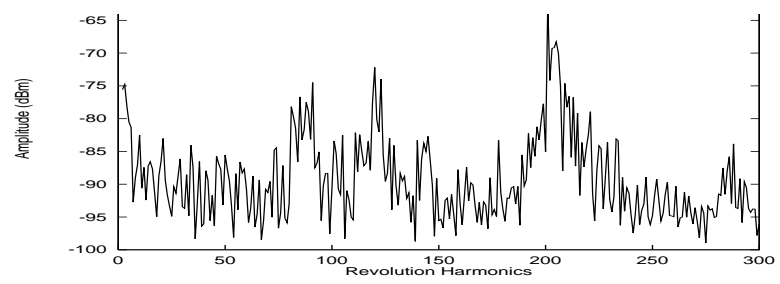

Figure 4: Measurement of vertical slow sideband for all of buckets filled at $1.3 \mathrm{GeV}$ and $138 \mathrm{~mA}$ in the normal operation vacuum pressure.

creased by increasing the beam energy, diluting the beam size or reducing the trapped ion density from the improvement on vacuum, the instability from ions could be dismissed. The shaking method knock out the ions by driving the beam oscillated at some particular frequency. The electron bunch is also redistributed from shaking. The electron density within bunches becomes smaller and less trapping potential from electron beam resulted. Ion produced instability is also reduced from this bunch redistribution process.

The damping mechanism from chromaticities complete the instability. If chromaticities are strong enough, the instability from ion driven force could be eliminated. From the transverse spectrum data, the coherent peaks of $\mathrm{H}_{2}^{+}$oscillation frequency show up first as the ion force cause instability. While as the ion driven force getting larger, additional peaks appear from the indication of venting $\mathrm{H}_{2}$ gas experiment. This feature can't be fully explained from conventional ion and fast beam ion theory. The impedance like behavior was suggested to explain this phenomenon. While more works would be down in the future to verify this assumption.

\section{REFERENCES}

[1] J. C. Lee et al., Proceedings of the European Particle Accelerator Conference, p1159, 1994.

[2] J. C. Lee et al., Investigation of Ion Effects in SRRC Storage Ring by Venting $\mathrm{H}_{2}$ gas, This Proceedings.

[3] J. C. Lee et al., Proceedings of the Particle Accelerator Conference, p2968, 1995.

[4] M. H. Wang et al., Experiment of RF Voltage Modulation at SRRC, This Proceedings.

[5] G. H. Luo et al., Proceedings of the Particle Accelerator Conference, p853, 1997.

[6] Y. J. Hsu et al. , Proceedings of the European Particle Accelerator Conference, p2175, 1998.

[7] T. O. Raubenheimer and F. Zimmermann, Phys. Rev. E 52, p5487, 1995.

[8] J. Byrd et al., Phys. Rev. Lett., p79, 1997.

[9] J. Y. Huang et al., Phys. Rev. Lett., p4388, 1998. 